\title{
The clamped-free rod under inclined end forces and transitions between equilibrium configurations
}

\author{
P. Singh • V. G. A. Goss
}

Received: 28 January 2019 / Accepted: 26 July 2019 / Published online: 16 August 2019

(C) The Author(s) 2019

\begin{abstract}
We investigate the problem of the straight, inextensible and unshearable clamped-free elastic rod subjected to an inclined end force. Exact analytic solutions representing all equilibrium configurations of the deformed rod are presented in elliptic integral form. Those exact solutions, for a given angle of inclination of the end force and number of inflection points, are characterised by two quantities; the end force and the elliptic modulus. Critical points are discussed and analytic conditions for determining their location are presented. Certain critical points where transitions between two equilibrium configurations whose numbers of inflection points differs by one are pointed out. Simple formulae for the total number of equilibrium configurations for a given end force are given. Applying arguments based on the elastic strain energy of the rod, we discuss scenarios where highly inflectional equilibrium configurations can transition to equilibrium configurations with fewer inflection points.
\end{abstract}

Keywords Clamped-free - Elastica · Elliptic integrals · Equilibrium configurations · Inclined end forces · Transitions

\section{Introduction}

Large deformations of clamped-free rods, also known as cantilevers, has been the subject of study by many researchers. For a complete and comprehensive historical review of this subject, we refer the readers to the works of Navaee and Elling [1] and Batista [2] and the references cited therein.

The clamped-free rod under a purely compressive end force succumbs to large post-buckling deformations once the rod has buckled under a critical force. The character of the large post-buckling deformations of the rod will depend on the mode $n$ the rod has buckled into, where $n=1,2,3, \ldots$, see Timoshenko and Gere [3]. Those modes $n$ reflect the number of inflection points present in an exact solution representing an equilibrium configuration for large post-buckling deformations and also provide a means for distinguishing or classifying those exact solutions.

This article revisits the problem of determining exact solutions for all equilibrium configurations of the clampedfree rod under inclined end forces. The inclined end force is provided to the rod in such a manner that even

P. Singh $(\varangle)$ • V. G. A. Goss

School of Engineering, London South Bank University, London SE1 OAA, UK

e-mail: singhp9@1sbu.ac.uk

V. G. A. Goss

e-mail: gossga@1sbu.ac.uk 
though the force increases, the angle of inclination of the force is kept constant. The angle of inclination of the end force is restricted to an interval which allows the discussion of the clamped-free rod under compression, the pure cantilever, and all other cases in between in a single unified approach. Although that is an old problem and many of the results are well known — see Navaee and Elling [1,4] and Batista [2,5] — this paper draws attention to certain aspects of the mechanics of the problem that have been overlooked. Firstly, transitions and jumps can occur at critical points between equilibrium configurations with different numbers of inflection points when increasing and decreasing the end force, i.e. loading and unloading the rod, respectively. Secondly, the manifestation of those transitions and jumps depends on which parameter is being controlled during loading and unloading the rod; for example, control of the end force (dead loading) leads to different transitions from control of end displacement (rigid loading) [6]. Thirdly, transitions and jumps between equilibrium configurations with different numbers of inflection points involve changes in the elastic strain energy of the rod. That observation offers a means of understanding which equilibrium configuration is selected when a rod undergoes a transition or jump. We apply that elastic strain energy-based argument to explain how a rod in a highly inflectional equilibrium configuration can transition to an equilibrium configuration with fewer inflection points, and ultimately the equilibrium configuration representing the straight rod, by releasing elastic strain energy.

In our analysis of the aforementioned transitions and jumps, we first determine exact solutions for all equilibrium configurations of the clamped-free rod subjected to an inclined end force in terms of elliptic integrals. Those exact solutions are presented in a form that makes their dependency on the number of inflection points explicit. We can then classify exact solutions representing equilibrium configurations by the number of inflection points, as for the case of the rod under pure compression. That is very useful for counting the number of equilibrium configurations for a given end force. We show that the exact solution representing each equilibrium configuration for a given angle of inclination of the end force and number of inflection points can be characterised by two quantities: the end force and the elliptic modulus (a function of the angle at the free end). Consequently, once we have determined those quantities, the shape of the deformed rod is reduced to a simple exercise in plotting points with elliptic integrals.

For each equilibrium configuration of the clamped-free rod under an inclined end force, at any inflection point, we give the slope or angle, the arc length parameter along the rod, and the coordinates. We additionally discuss critical points and present analytic conditions for determining their location. Furthermore, we show that the local force minima (critical points) can be used to define ranges for the values of inclined end force between which the number of inflectional equilibrium configurations can be counted. Simple formulae for the number of equilibrium configurations for a given end force are thereby determined. Finally, we use the results of our analysis to examine how transitions and jumps between equilibrium configurations with different numbers of inflection points can arise.

\section{Mathematical model}

We consider an elastic rod parameterised by its non-dimensional (normalised) arc length, $0 \leq s \leq 1$. In the Cartesian coordinate frame with coordinates $(x, y)$, the rod is clamped at the origin $(s=0)$ and free at the other end $(s=1)$. The rod is initially straight, inextensible and unshearable but can deform in the $x y$-plane when the free end is subjected to a non-dimensional force $f$ inclined at angle $\alpha$. The angle the tangent at any point $(x(s), y(s))$ on the deformed rod makes with the $x$-axis is given by $\theta(s)$, see Fig. 1 . The (non-dimensional) force $f$ is positive and the angle $\alpha$ is restricted to $0 \leq \alpha \leq \pi$. There is no need to consider values of $\alpha$ in $\pi<\alpha \leq 2 \pi$, due to symmetry.

In the Cartesian coordinate frame with coordinates $(u, v)$, which is rotated clockwise through angle $\alpha$ with respect to the $(x, y)$ coordinate frame, as depicted in Fig. 1, we see that

$x=\cos (\alpha) u+\sin (\alpha) v$,

$y=-\sin (\alpha) u+\cos (\alpha) v$.

Furthermore, the angle the tangent at any point $(u(s), v(s))$ on the deformed rod makes with the $u$-axis is $\psi(s)$ given by

$\psi=\theta+\alpha$. 

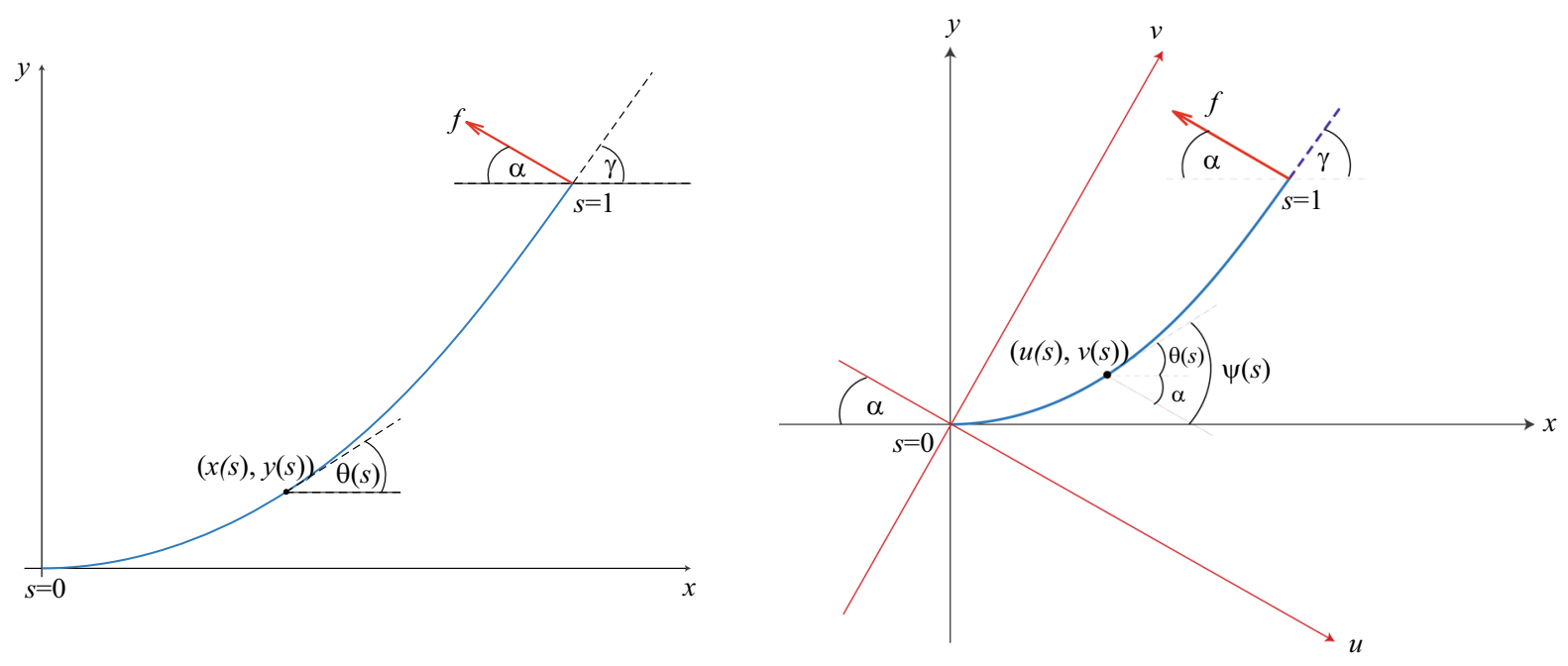

Fig. 1 The diagram on the left: the general configuration of the deformed rod with reference to the $(x, y)$ coordinate frame under an end force $f$ inclined at angle $\alpha$. The diagram on the right: the same deformed rod with reference to the $(u, v)$ coordinate frame rotated clockwise through angle $\alpha$ with respect to the $(x, y)$ coordinate frame. In the $(u, v)$ coordinate frame, the end force $f$ acts along the $u$-axis. Note, $\gamma=\theta(1)$. (Color figure online)

The (non-dimensional) ordinary differential equations describing the clamped-free rod, which may be derived from force and moment balance arguments applied to an infinitesimal element of deformed rod, are

$$
\begin{aligned}
\frac{\mathrm{d}^{2} \psi}{\mathrm{d} s^{2}} & =-f \sin (\psi), \\
\frac{\mathrm{d} u}{\mathrm{~d} s} & =\cos (\psi), \\
\frac{\mathrm{d} v}{\mathrm{~d} s} & =\sin (\psi) .
\end{aligned}
$$

The boundary conditions are

$$
\begin{aligned}
\psi(0) & =\alpha, \\
\frac{\mathrm{d} \psi(1)}{\mathrm{d} s} & =0, \\
u(0) & =0, \\
v(0) & =0 .
\end{aligned}
$$

Once $\psi, u$ and $v$ are known, it is easy to determine $\theta, x$ and $y$ from Eqs. (1), (2) and (3). Apart from the convenience of not having to carry around all those factors of $\cos (\alpha)$ and $\sin (\alpha)$ in our calculations, another reason for working in the $(u, v)$ coordinate frame is that the inclined end force $f$ acts along the $u$-axis.

\section{Exact solutions}

When $\alpha=0$, we have the rod under pure compression; for $\alpha=\pi / 2$, we have the pure cantilever; and at $\alpha=\pi$, we have the rod under pure tension. We will consider the case $\alpha=\pi$ first, since the rod is inextensible, and this case is the simplest to deal with. The case $\alpha=0$ is dealt with last, as this can be obtained by taking the $\alpha \rightarrow 0$ limit in the case $0<\alpha<\pi$. 
$3.1 \alpha=\pi$

The inextensibility of the rod restricts the exact solutions available. The obvious solution is

$\theta=0, \quad x=s, \quad y=0$.

The above solution represents a rod that remains straight under all $f \geq 0$, see Antman [7]. That case was not discussed by Navaee and Elling [1]; however, Batista mentions it briefly [2]. We mention it here for the sake of completeness, but will not discuss it any further.

\section{$3.20<\alpha<\pi$}

Integrating Eq. (4) subject to the boundary condition in Eq. (8), we find

$\frac{\mathrm{d} \psi}{\mathrm{d} s}= \pm 2 f^{1 / 2}\left(k^{2}-\sin ^{2}\left(\frac{\psi}{2}\right)\right)^{1 / 2}$

where, anticipating elliptic integrals, we introduce the elliptic modulus $k$ given by

$k=\sin \left(\frac{\gamma+\alpha}{2}\right) \Leftrightarrow \gamma=2 \arcsin (k)-\alpha$

and $\gamma=\theta(1)$. Note that we have taken $f$ to be a constant during the integration procedure.

Equilibrium configurations of the deformed rod may have $n$ inflection points, where $n=1,2,3, \ldots$. At an inflection point $\mathrm{d} \theta / \mathrm{d} s=\mathrm{d} \psi / \mathrm{d} s=0$, so from Eqs. (12) and (13), for an equilibrium configuration with $n$ inflection points, the $i$ th inflection point is at

$\psi_{i}=\psi\left(s_{i}\right)=(-1)^{n+i}(\gamma+\alpha)$,

where $s_{i}$ is the value of $s$ at the $i$ th inflection point and $i=1,2,3, \ldots, n$. We have $s_{0} \leq s_{1}<s_{2}<s_{3}<\cdots<s_{n}$. Note that $s_{n}=1$, and for notational convenience, we set $s_{0}=0$ and clarify that the latter is not an inflection point. The sign in Eq. (14) is chosen to ensure that the angle at the free end of the rod, where there is an inflection point (see Eq. (8)), is $\psi_{n}=\psi\left(s_{n}\right)=\psi(1)=\gamma+\alpha$.

Solutions to Eq. (12) exist if $k^{2}-\sin ^{2}(\psi / 2) \geq 0$. That condition places restrictions on the allowed values of $\gamma$. Under the inclined end force $f$ the rod deforms with either $\gamma \geq 0$ or $\gamma<0$. As $f \rightarrow \infty$, in the former case, we will have $\gamma \rightarrow \pi-\alpha$, and in the latter case, $\gamma \rightarrow-(\pi+\alpha)$. To explain this, observe when $f \neq 0$, Eq. (4) can be re-written as $(1 / f)\left(\mathrm{d}^{2} \psi / \mathrm{d} s^{2}\right)+\sin (\psi)=0$. As $f \rightarrow \infty$, the dominant term is $\sin (\psi)$. Thus, we get $\sin (\psi)=0$. For the inflectional solutions considered in this paper, we obtain $\psi= \pm \pi$. That solution is valid near $s=1$ since the boundary condition at $s=1$, Eq. (8), holds for $\psi= \pm \pi$. So we can set $s=1$ in that solution and obtain $\gamma+\alpha= \pm \pi$ as required. Note that $\psi= \pm \pi$ does not satisfy the boundary condition at $s=0$, given by Eq. (7). At $s=0$, we have a boundary layer, i.e. a concentration of curvature, which changes the orientation of the rod at $s=0$ from $\psi(0)=\alpha$ to $\psi(0)= \pm \pi$. See Audoly and Pomeau [8] for a discussion on the method behind deriving the solution $\psi= \pm \pi$ as $f \rightarrow \infty$ and boundary layers. When $0 \leq \gamma<\pi-\alpha, k^{2}-\sin ^{2}(\psi / 2) \geq 0$ always holds. When $-(\pi+\alpha)<\gamma<0, k^{2}-\sin ^{2}(\psi / 2) \geq 0$ only holds when $\gamma \leq-2 \alpha$. Hence, the negative and positive values of $\gamma$ must be restricted to the intervals $-(\pi+\alpha)<\gamma \leq-2 \alpha$ and $0 \leq \gamma<\pi-\alpha$, respectively. We refine those restrictions slightly to conform with describing equilibrium configurations by the number of inflection points $n$. We require

$\begin{array}{lll}-(\pi+\alpha)<\gamma<-2 \alpha, & 0 \leq \gamma<\pi-\alpha, & n=1,3,5, \ldots \\ -(\pi+\alpha)<\gamma \leq-2 \alpha, & 0<\gamma<\pi-\alpha, & n=2,4,6, \ldots\end{array}$ 
Our derivations, based on an examination of plots of $\sin ^{2}(\psi / 2)$, will not be elaborated on here since the conditions given by Eqs. (15) and (16) have been presented by Navaee and Elling [4]. The implications of Eqs. (15) and (16) for the elliptic modulus $k$ are

$$
\begin{aligned}
& -1<k<-\sin \left(\frac{\alpha}{2}\right), \quad \sin \left(\frac{\alpha}{2}\right) \leq k<1, \quad n=1,3,5, \ldots, \\
& -1<k \leq-\sin \left(\frac{\alpha}{2}\right), \quad \sin \left(\frac{\alpha}{2}\right)<k<1, \quad n=2,4,6, \ldots
\end{aligned}
$$

In order to completely determine $\psi, u$ and $v$, introduce

$k \sin (\phi)=\sin \left(\frac{\psi}{2}\right)$

and follow the approach similar to the one outlined by Frisch-Fay [9] or Dym [10]. Since $\psi / 2$ always lies between $-(\gamma+\alpha) / 2$ and $(\gamma+\alpha) / 2, \cos (\psi / 2)>0$. Thus, we can differentiate Eq. (19) with respect to $\psi$ and obtain

$\frac{\mathrm{d} \phi}{\mathrm{d} \psi}=\frac{\left(1-k^{2} \sin ^{2}(\phi)\right)^{1 / 2}}{2 k \cos (\phi)}$.

Also, from Eq. (19), we have

$\phi=\arcsin \left(\frac{\sin (\psi / 2)}{k}\right)$,

where $\phi$ is the elliptic argument. The value of $\phi$ at $\psi=\alpha$ is denoted by

$\phi_{\alpha}=\arcsin \left(\frac{\sin (\alpha / 2)}{k}\right)$,

and the value of $\phi$ at $\psi=\psi_{i}$ is denoted by

$\phi_{i}=(-1)^{n+i} \frac{\pi}{2}$,

for $i=1,2,3, \ldots, n$. Combining Eq. (12) with the correct sign and Eq. (20), we find for $s_{i-1}<s<s_{i}$,

$\frac{\mathrm{d} \phi}{\mathrm{d} s}=(-1)^{n+i} f^{1 / 2}\left(1-k^{2} \sin ^{2}(\phi)\right)^{1 / 2}$

for $i=1,2,3, \ldots, n$. It's a straightforward procedure to use Eqs. (5), (6), (19) and (21)-(24) to calculate, for $s_{i-1} \leq s \leq s_{i}$

$s=\frac{1}{f^{1 / 2}}\left((-1)^{n+i} F(\phi, k)+(-1)^{n} F\left(\phi_{\alpha}, k\right)+2(i-1) K(k)\right)$,

$u=\frac{1}{f^{1 / 2}}\left((-1)^{n+i}(2 E(\phi, k)-F(\phi, k))+(-1)^{n}\left(2 E\left(\phi_{\alpha}, k\right)-F\left(\phi_{\alpha}, k\right)\right)+2(i-1)(2 E(k)-K(k))\right)$,

$v=\frac{2 k}{f^{1 / 2}}\left(-(-1)^{n+i} \cos (\phi)+(-1)^{n+1} \cos \left(\phi_{\alpha}\right)\right)$

for $i=1,2,3, \ldots, n . K(k)$ and $E(k)$ are the complete elliptic integrals of the first and second kind, respectively. $F(\phi, k)$ and $E(\phi, k)$ are the incomplete elliptic integrals of the first and second kind, respectively. See Gradshteyn and Ryzhik [11] for further details on elliptic integrals.

The values of $s, u$ and $v$ at the $i$ th inflection point are

$$
\begin{aligned}
s_{i} & =\frac{1}{f^{1 / 2}}\left((-1)^{n} F\left(\phi_{\alpha}, k\right)+(2 i-1) K(k)\right), \\
u_{i} & =\frac{1}{f^{1 / 2}}\left((-1)^{n}\left(2 E\left(\phi_{\alpha}, k\right)-F\left(\phi_{\alpha}, k\right)\right)+(2 i-1)(2 E(k)-K(k))\right), \\
v_{i} & =(-1)^{n+1} \frac{2 k}{f^{1 / 2}} \cos \left(\phi_{\alpha}\right),
\end{aligned}
$$


where $u_{i}=u\left(s_{i}\right), v_{i}=v\left(s_{i}\right)$ and $i=1,2,3, \ldots, n$. The values of $s, u$ and $v$ at the $n$th (last) inflection point, where $s=s_{n}=1$, are particularly important so we state them explicitly

$$
\begin{aligned}
1 & =\frac{1}{f^{1 / 2}}\left((-1)^{n} F\left(\phi_{\alpha}, k\right)+(2 n-1) K(k)\right), \\
u(1) & =\frac{1}{f^{1 / 2}}\left((-1)^{n}\left(2 E\left(\phi_{\alpha}, k\right)-F\left(\phi_{\alpha}, k\right)\right)+(2 n-1)(2 E(k)-K(k))\right), \\
v(1) & =(-1)^{n+1} \frac{2 k}{f^{1 / 2}} \cos \left(\phi_{\alpha}\right),
\end{aligned}
$$

where $u_{n}=u\left(s_{n}\right)=u(1)$ and $v_{n}=v\left(s_{n}\right)=v(1)$. A solution, $\psi, u$ and $v$, describing an equilibrium configuration of the rod is completely determined once $f$ and $k$ are known. The rod is deformed by providing an inclined force $f$ applied to the free end of the rod, i.e. we input values of $f$ into Eq. (31) and numerically solve for corresponding values of the elliptic modulus $k$. However, it is much simpler to input the allowed values of $k$ given by Eqs. (17) and (18) into Eq. (31) and obtain the corresponding values for $f$ from which force-elliptic modulus $(f-k)$ plots can be obtained. Determining the $x y$-shape of the deformed rod is now a simple exercise in plotting points with elliptic integrals using Eqs. (1), (2) and (25)-(30).

It may be noticed that for a given $n$, solving Eq. (31) for a specified $f$ could result in more than one value for $k$, since $k$ can be positive (when $\gamma>0$ ) or negative (when $\gamma<-2 \alpha$ ). Thus, for a given $n$ and a specified $f$, we expect to have non-uniqueness, i.e. multiple solutions. See Sect. 4.1 for a more detailed discussion of non-uniqueness.

\section{$3.3 \alpha=0$}

When $\alpha=0$, we have $\theta=\psi, x=u, y=v$ and the results given by Eqs. (25)-(33) above reduce to the solutions for deformations of the clamped-free rod from the $n$th buckled mode. As for the case with $0<\alpha<\pi$, a solution $\theta, x$ and $y$, describing an equilibrium configuration of the rod is completely determined once $f$ and $k$ are known. The allowed values of $k$ are given by setting $\alpha=0$ in Eqs. (17) and (18). Explicitly, we find

$-1<k<1$.

Input those values of $k$ into Eq. (31), where we have already set $\alpha=0$, and obtain the corresponding values for $f$.

\section{Properties of the equilibrium configurations}

The rod is deformed by providing an inclined force $f$ applied to the free end of the rod. In that case, we can interpret all physical quantities as being functions of $f$, for example, $k=k(f)$.

As mentioned above, the case $\alpha=\pi$ only has the solution given by Eq. (11) for all $f \geq 0$. Therefore, we only consider the $0<\alpha<\pi$ and $\alpha=0$.

\section{$4.10<\alpha<\pi$}

If we take $k=k(f)$, the critical points on $f-k$ plots are determined from $\mathrm{d} k / \mathrm{d} f=0$ and $\mathrm{d} f / \mathrm{d} k=0$, which can be easily found from Eq. (31). Taking $\mathrm{d} k / \mathrm{d} f=0$, we find

$k= \begin{cases}-\sin (\alpha / 2), & n=2,4,6, \ldots, \\ \sin (\alpha / 2), & n=1,3,5, \ldots,\end{cases}$

where the corresponding values of $f$ denoted by $f_{\alpha}^{(n)}$ are

$f_{\alpha}^{(n)}=4(n-1)^{2} K^{2}\left(\sin \left(\frac{\alpha}{2}\right)\right)$. 

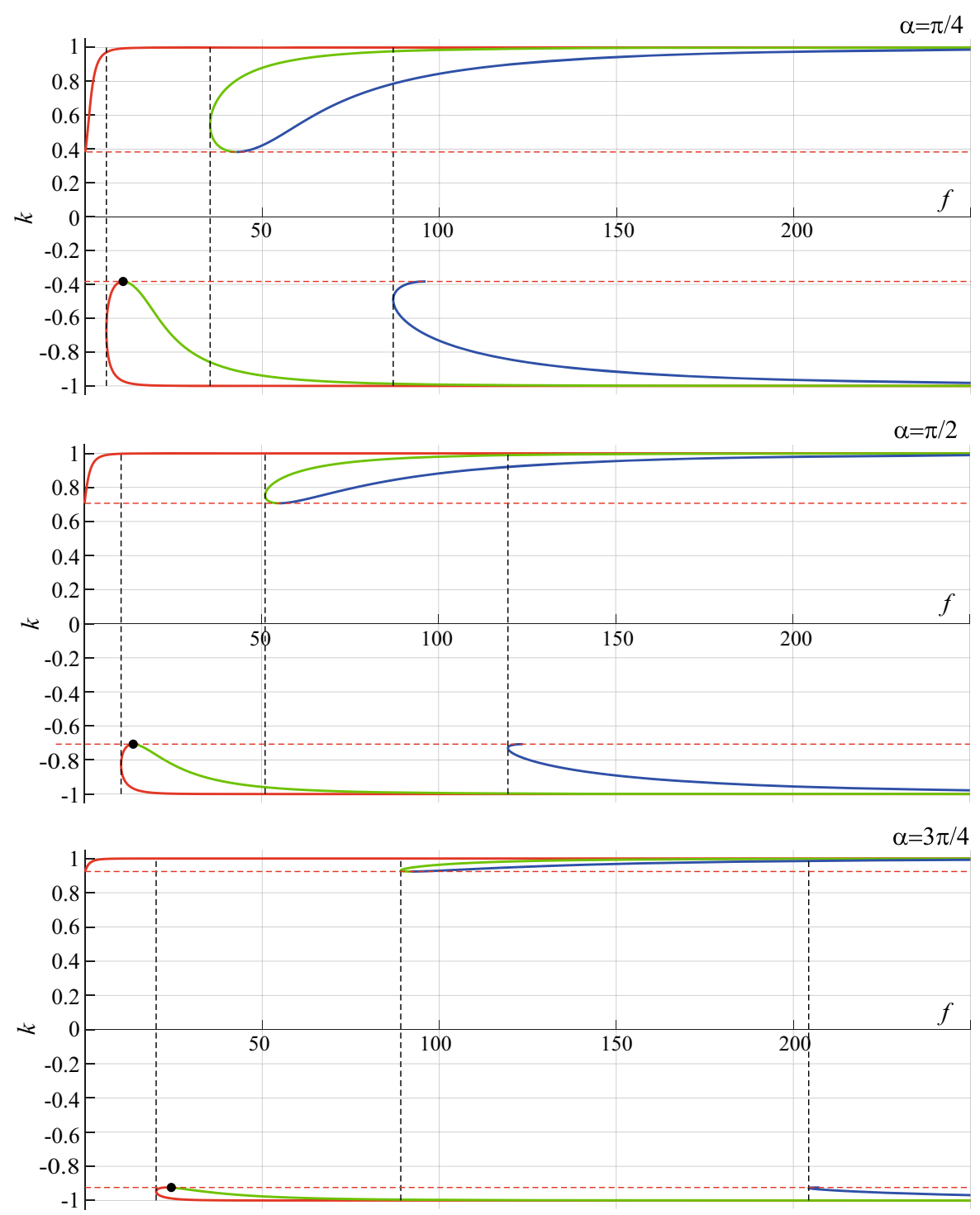

Fig. $2 f-k$ plots for equilibrium configurations with 1 (red), 2 (green) and 3 (blue) inflection points for $\alpha=\pi / 4, \pi / 2$ and $3 \pi / 4$. (Color figure online)

Those critical points are associated with local minima and maxima for $k$. Setting $\mathrm{d} f / \mathrm{d} k=0$, gives

$\frac{(-1)^{n} \operatorname{sgn}(k) \sin (\alpha)}{2\left(k^{2}-\sin ^{2}(\alpha / 2)\right)^{1 / 2}}-(-1)^{n}\left(E\left(\phi_{\alpha}, k\right)-\left(1-k^{2}\right) F\left(\phi_{\alpha}, k\right)\right)-(2 n-1)\left(E(k)-\left(1-k^{2}\right) K(k)\right)=0$,

where $\operatorname{sgn}(k)$ denotes the sign of $k$. Equation (37) can be solved for $k=k_{\min }^{(n)}$ for $-1<k_{\min }^{(n)}<-\sin (\alpha / 2)$ when $n=1,3,5, \ldots$ and for $\sin (\alpha / 2)<k_{\min }^{(n)}<1$ when $n=2,4,6, \ldots$ Substitute $k=k_{\min }^{(n)}$ into Eq. (31) to find the corresponding value of $f$ which we denote by $f_{\min }^{(n)}$. Those critical points are associated with local minima for $f$. That can be seen by simply rotating the $f-k$ plots in Fig. 2 such that the $f$-axes point vertically up. Batista presents a version of Eq. (37) in terms of Jacobi elliptic functions, but only solves for $n=1$ and $-1<k<-\sin (\alpha / 2)$ [5]. In that condition, unlike our condition given by Eq. (37), the dependency on the number of inflection points $n$ is not explicit. 
We illustrate our results for $\alpha=\pi / 4, \pi / 2$ and $3 \pi / 4$ with $f-k$ plots in Fig. 2 . In that figure, the vertical black dashed lines pass through the critical points $\left(f_{\min }^{(n)}, k_{\min }^{(n)}\right)$ for $n=1,2,3, \ldots$ The horizontal red dashed lines in that figure pass through the critical points $\left(f_{\alpha}^{(n)},-\sin (\alpha / 2)\right)$ for $n=2,4,6, \ldots$ and $\left(f_{\alpha}^{(n)}, \sin (\alpha / 2)\right)$ for $n=1,3,5, \ldots$. At those critical points, for $n \geq 2$, transitions between equilibrium configurations with $n$ and $n-1$ inflection points are possible under increasing and decreasing $f$, i.e. loading and unloading the rod, respectively. For example, at $\left(f_{\alpha}^{(2)},-\sin \left(\frac{\alpha}{2}\right)\right)$, marked as a black dot on each of the plots in Fig. 2, the rod may deform into an equilibrium configuration with either 1 inflection point, if $f$ decreases, or 2 inflection points, if $f$ increases.

The conditions for critical points of any other physical quantity, such as $y(1)=-\sin (\alpha) u(1)+\cos (\alpha) v(1)$ can be determined from $\mathrm{d} y(1) / \mathrm{d} f=-\sin (\alpha)(\mathrm{d} u(1) / \mathrm{d} f)+\cos (\alpha)(\mathrm{d} v(1) / \mathrm{d} f)=0$ and $\mathrm{d} f / \mathrm{d} y(1)=0$ following the procedure just described for $k=k(f)$.

As mentioned at the end of Sect. 3.2, we expect multiple solutions for a specified $f$, i.e. non-uniqueness. Consider solutions for a given $n$. When we specify $f$ and look for solutions to Eq. (31), we will find solutions where $k$ could be positive or negative, corresponding to deformations of the rod into equilibrium configurations with positive or negative end angle $\gamma$, respectively. On an intuitive level, that makes sense since we can deform the rod in two different directions $(\gamma<-2 \alpha$ or $\gamma>0)$ and therefore we can reasonably expect multiple solutions for the same $f$. Even so, there are still two properties which affect non-uniqueness. Firstly, the asymmetry between deforming the rod with $\gamma<-2 \alpha(k<-\sin (\alpha / 2))$ and $\gamma>0(k>\sin (\alpha / 2))$ results in two different domains for $f$. To see that, compare plots for a given $n$ with $\gamma<-2 \alpha(k<-\sin (\alpha / 2))$ and $\gamma>0(k>\sin (\alpha / 2))$ in Fig. 2 . When the two domains for $f$ overlap, we have multiple solutions (non-uniqueness), when there is no overlap, we have one solution (uniqueness). Secondly, the presence of local minima for $f$ at $\left(f_{\min }^{(n)}, k_{\min }^{(n)}\right)$ means that for $f$ such that $f_{\min }^{(n)}<f<f_{\alpha}^{(n+1)}$, we can have two solutions for $k$, each on either side of $k_{\min }^{(n)}$ and with the same sign as $k_{\min }^{(n)}$, see Fig. 2. We can summarise all that information in a single equation. Let $N_{\mathrm{e}}(n, f)$ denote the number of equilibrium configurations for a given $n=1,2,3, \ldots$ and specified $f$, then

$N_{\mathrm{e}}(n, f)= \begin{cases}1, & f_{\alpha}^{(n)} \leq f<f_{\min }^{(n)}, \\ 2, & f=f_{\min }^{(n)}, \\ 3, & f_{\min }^{(n)}<f<f_{\alpha}^{(n+1)} \\ 2, & f \geq f_{\alpha}^{(n+1)}\end{cases}$

If we denote the number of equilibrium configurations for a given $f$ by $N_{\mathrm{e}}(f)$, then

$$
N_{\mathrm{e}}(f)= \begin{cases}1, & 0 \leq f<f_{\min }^{(1)}, \\ 2 n, & f=f_{\min }^{(n)}, \\ 2 n+1, & f_{\min }^{(n)}<f<f_{\min }^{(n+1)}\end{cases}
$$

for $n=1,2,3, \ldots$. An illustration of Eq.hus (39) is provided by Fig. 3.

With multiple solutions representing the equilibrium configurations of the rod for a given $f$, which solution is selected? According to Love, solutions with internal inflection points are unstable [12]. Therefore, only the solutions for $n=1$ are stable. Batista also holds that view and adds that the solution for $n=1$ is also unstable for the section of curve between points $\left(f_{\alpha}^{(2)},-\sin (\alpha / 2)\right)$ and $\left(f_{\min }^{(1)}, k_{\min }^{(1)}\right)$ on any of the plots in Fig. 2 [5]. Everywhere else, the solution for $n=1$ is stable. If stability is the criteria for selecting a solution for a given $f$, then solutions with $n=1$ are likely to be selected. However, equilibrium configurations for higher $n=2,3, \ldots$ although unstable, may be realised in the presence of suitable constraints applied at certain inflection points that suppress equilibrium configurations with lower $n$. In Bigoni et al, those constraints are referred to as "clamped-guided" [13]. 


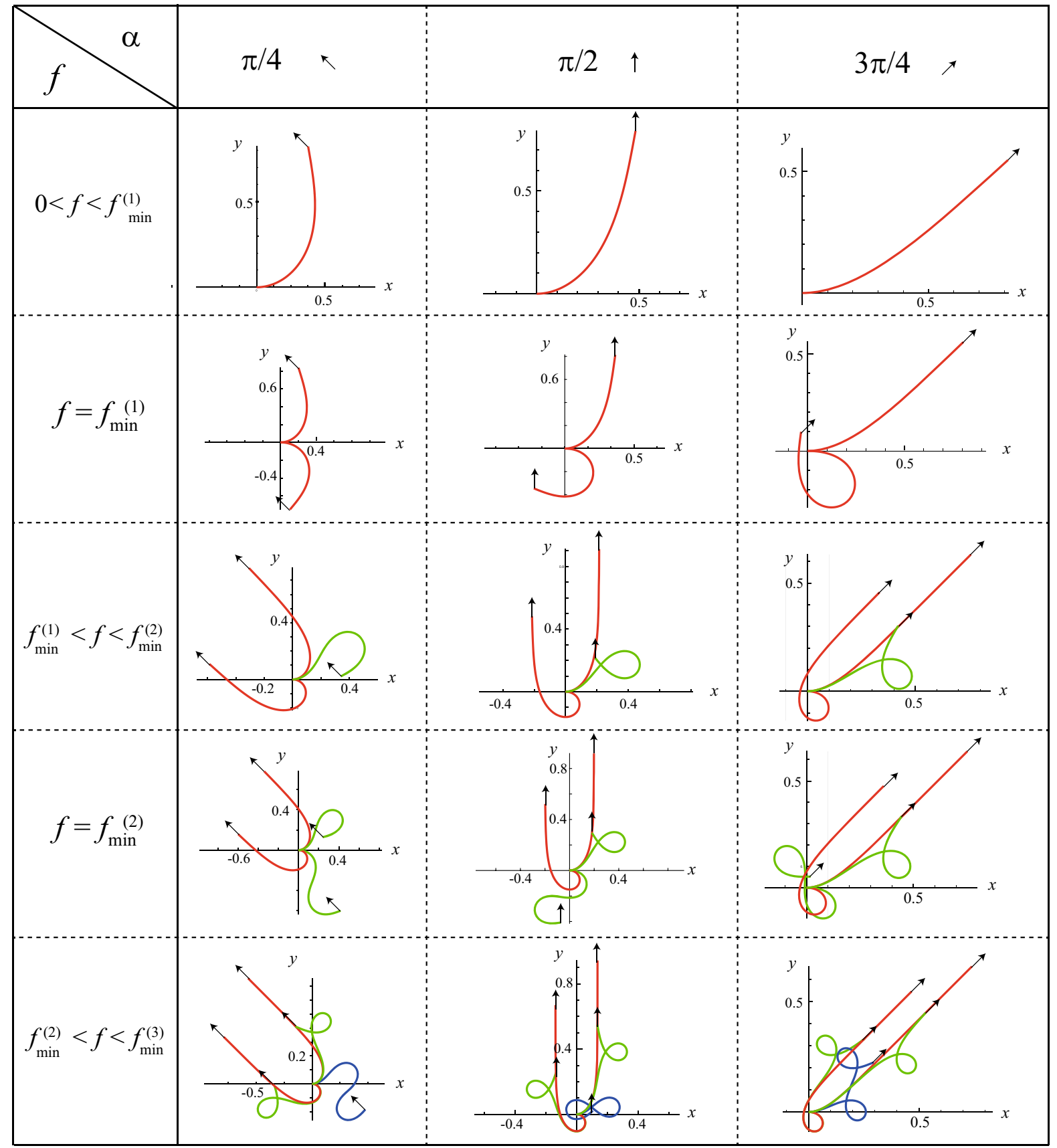

Fig. 3 The $x-y$ shapes for equilibrium configuration with 1 (red), 2 (green) and 3 (blue) inflection points for $\alpha=\pi / 4, \pi / 2$ and $3 \pi / 4$ and various values of $f$. (Color figure online)

$4.2 \alpha=0$

The critical force, denoted by $f_{\mathrm{c}}^{(n)}$, at which the rod will buckle into the $n$th mode can be found by setting $k=0$ in Eq. (31) where we have already set $\alpha=0$. We find

$f_{\mathrm{c}}^{(n)}=\frac{(2 n-1)^{2}}{4} \pi^{2}$. 


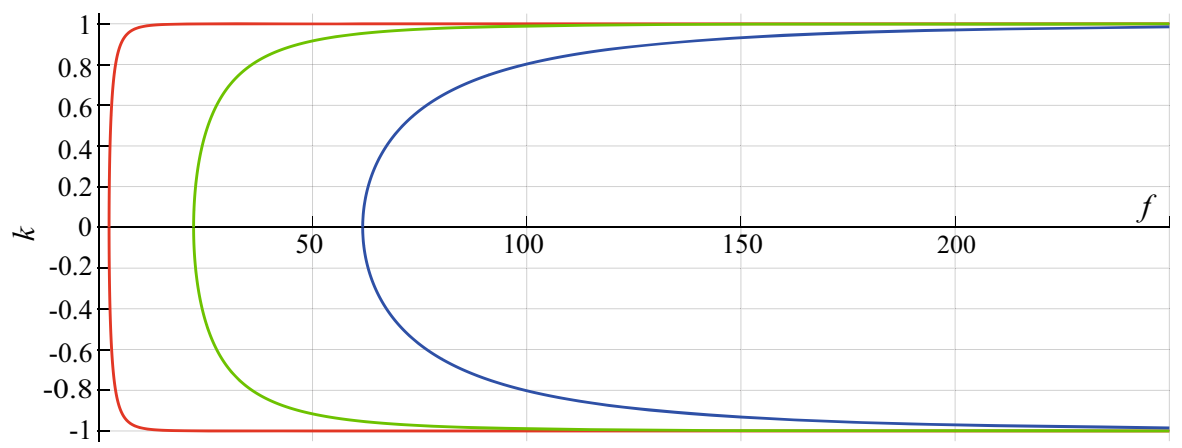

Fig. $4 f-k$ plots for equilibrium configurations with 1 (red), 2 (green) and 3 (blue) inflection points when $\alpha=0$. (Color figure online)

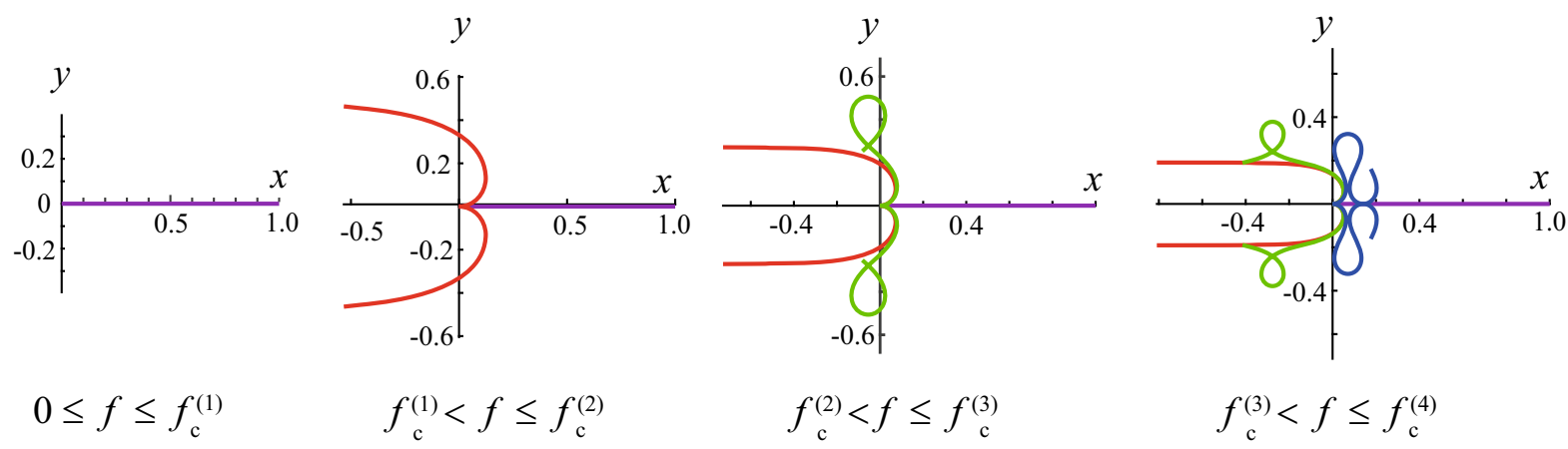

Fig. 5 The $x-y$ shapes for equilibrium configuration with 1 (red), 2 (green) and 3 (blue) inflection points for $\alpha=0$ and various values of $f$. The straight rod is represented by magenta. (Color figure online)

Incidentally, since the critical force is the minimum force at which the rod deforms, see Fig. 4, it can also be obtained by setting $\alpha=0$ in Eq. (37) to obtain the condition

$E(k)-\left(1-k^{2}\right) K(k)=0$.

The solution to Eq. (41) is $k=0$. Substitute that value of $k$ into Eq. (31) to find $f_{\min }^{(n)}=f_{\mathrm{c}}^{(n)}$. In effect, we have demonstrated that

$\lim _{\alpha \rightarrow 0} f_{\min }^{(n)}=f_{\mathrm{c}}^{(n)}$

as expected.

Let $N_{\mathrm{e}}(n, f)$ denote the number of equilibrium configurations for a given $n=1,2,3, \ldots$ and specified $f$, then $N_{\mathrm{e}}(n, f)= \begin{cases}1, & 0 \leq f \leq f_{\mathrm{c}}^{(n)}, \\ 2, & f>f_{\mathrm{c}}^{(n)},\end{cases}$

and, if we denote the number of equilibrium configurations for a given $f$ by $N_{\mathrm{e}}(f)$, then

$N_{\mathrm{e}}(f)= \begin{cases}1, & 0 \leq f \leq f_{\mathrm{c}}^{(1)}, \\ 2 n+1, & f_{\mathrm{c}}^{(n)}<f \leq f_{\mathrm{c}}^{(n+1)}\end{cases}$

for $n=1,2,3, \ldots$. See Fig. 5 for an illustration of Eq. (44).

\section{Transitions between equilibrium configurations}

Increasing or decreasing the force $f$ in general will increase or decrease the elastic strain energy of the rod, respectively. Therefore, the elastic strain energy of the rod is important in any discussion where the force $f$ is varied. 


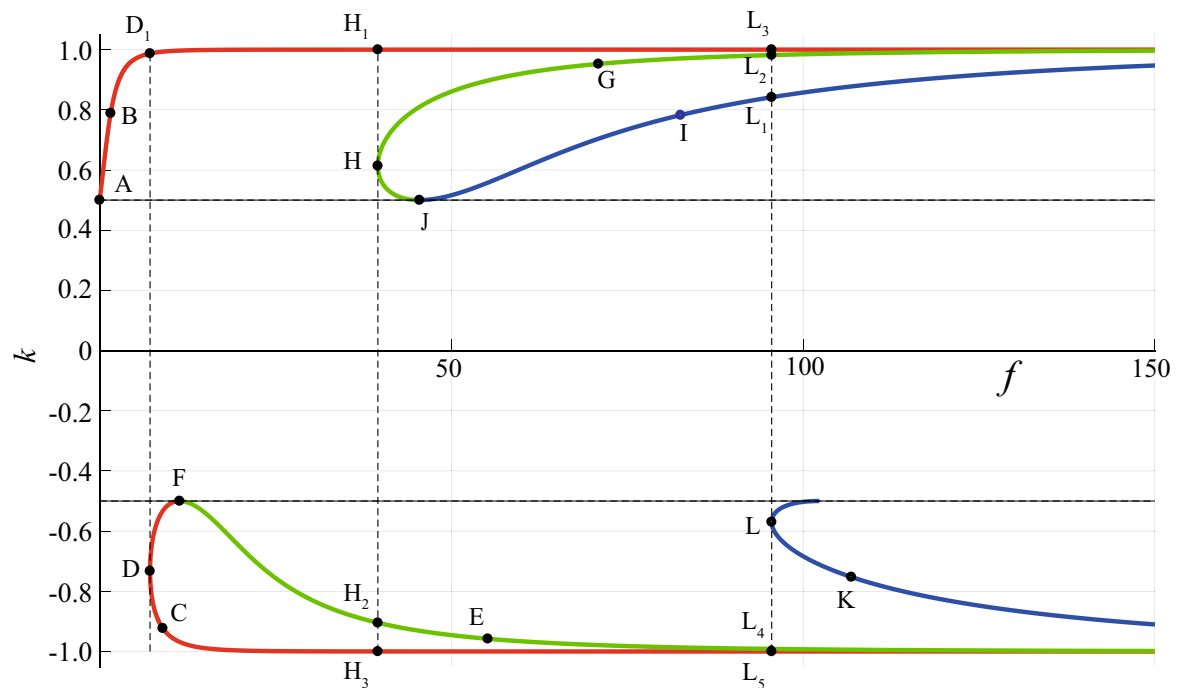

Fig. 6 A typical $f-k$ plot for equilibrium configurations with 1 (red), 2 (green) and 3 (blue) inflection points for $0<\alpha<\pi$. (Color figure online)

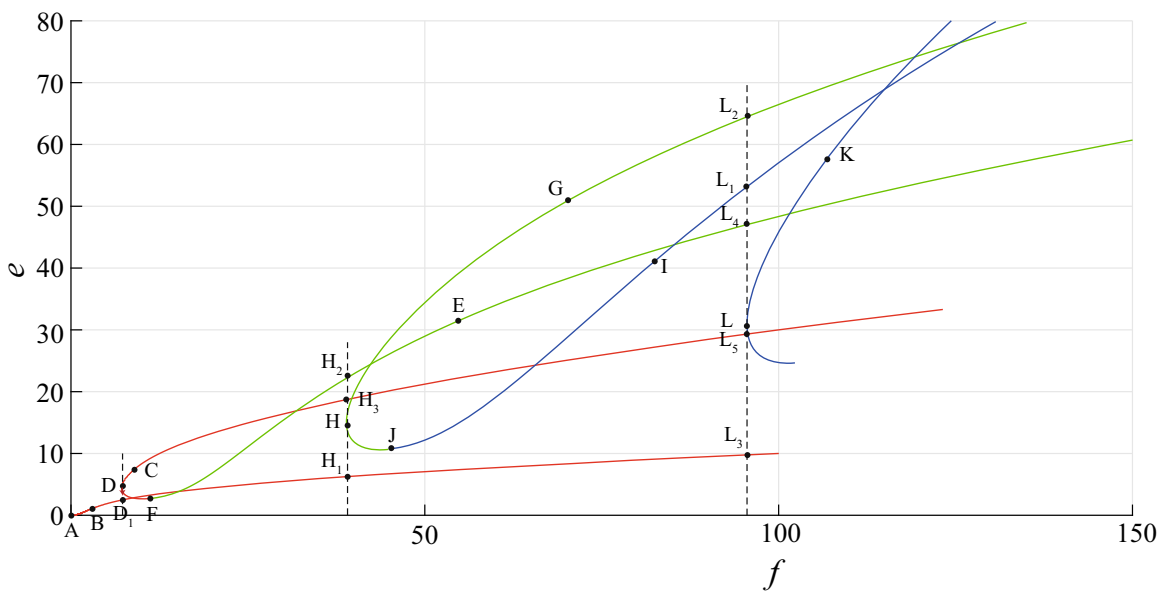

Fig. 7 A typical plot of $e$ against $f$ for equilibrium configurations with 1 (red), 2 (green) and 3 (blue) inflection points for $0<\alpha<\pi$. (Color figure online)

The (non-dimensional) elastic strain energy of the rod, $e$, is defined as

$e=\frac{1}{2} \int_{0}^{1} \mathrm{~d} s\left(\frac{\mathrm{d} \theta}{\mathrm{d} s}\right)^{2}=\frac{1}{2} \int_{0}^{1} \mathrm{~d} s\left(\frac{\mathrm{d} \psi}{\mathrm{d} s}\right)^{2}$

which after a short calculation becomes

$e=2 f^{1 / 2}\left((-1)^{n} E\left(\phi_{\alpha}, k\right)+(2 n-1) E(k)-\left(1-k^{2}\right)\left((-1)^{n} F\left(\phi_{\alpha}, k\right)+(2 n-1) K(k)\right)\right)$

for $n=1,2,3, \ldots$.

Consider the following scenarios:

1. The rod is in an equilibrium configuration with $n=1$ and $\gamma>0$ on the red curve denoted by B in Fig. 6 . Increasing $f$ will move to an equilibrium configuration higher up on the red curve and an increase in $e$, see Fig. 7. Decreasing $f$ moves to an equilibrium configuration lower down on the red curve and a decrease in $e$. Continually decreasing $f$ will result in a straight rod at A. 
2. The rod is in an equilibrium configuration with $n=1$ and $\gamma<-2 \alpha$ on the red curve denoted by $\mathrm{C}$ in Fig. 6. Increasing $f$ will move to an equilibrium configuration higher up on the red curve and an increase in $e$. Decreasing $f$ moves to an equilibrium configuration lower down on the red curve and a decrease in $e$. Continually decreasing $f$ will result in reaching the equilibrium configuration with $f=f_{\min }^{(1)}$ at $\mathrm{D}$. Once at $\mathrm{D}$, attempting to decrease $f$ further will result in a jump to $\mathrm{D}_{1}$, where $n=1$ and $\gamma>0$. At $\mathrm{D}_{1}$, decreasing $f$ proceeds as described in 1 .

3. The rod is in an equilibrium configuration with $n=2$ and $\gamma<-2 \alpha$ on the green curve denoted by $\mathrm{E}$ in Fig. 6. Increasing $f$ will move to an equilibrium configuration higher up on the green curve and an increase in $e$. Decreasing $f$ moves to an equilibrium configuration lower down on the green curve and a decrease in $e$. We can continue decreasing $f$ to reach $\mathrm{F}$, where $f=f_{\alpha}^{(2)}$. Decreasing $f$ further the equilibrium configuration transitions from one with $n=2$ and $\gamma=-2 \alpha$ to one with $n=1$ and $\gamma<-2 \alpha$. The furthest we can decrease $f$ to is $f=f_{\min }^{(1)}$ at $\mathrm{D}$. Once at $\mathrm{D}$, decreasing $f$ proceeds as described in 2. According to Love [12] and Batista [5] the section of curve between D and C is stable. However, we believe that section cannot be reached by starting on the green curve and continuously decreasing $f$. Our reasoning based on always keeping $e$ as small as possible will result in a path along the green curve that leads to $\mathrm{F}$, then $\mathrm{D}$, and finally proceeds as in 2 .

4. The rod is in an equilibrium configuration with $n=2$ and $\gamma>0$ on the green curve denoted by G in Fig. 6 . Increasing $f$ will move to an equilibrium configuration higher up on the green curve and an increase in $e$. Decreasing $f$ moves to an equilibrium configuration lower down on the green curve and a decrease in $e$. Continuously decreasing $f$ will result in reaching the equilibrium configuration with $f=f_{\min }^{(2)}$ at $\mathrm{H}$. Once at $\mathrm{H}$, attempting to decrease $f$ further will result in a jump. This time we have 3 choices to jump to: $\mathrm{H}_{1}, \mathrm{H}_{2}$ or $\mathrm{H}_{3}$. We argue that the process of decreasing $f$ removes work (energy) from the system which must be reflected in a corresponding decrease in $e$. Since $\mathrm{H}_{2}$ and $\mathrm{H}_{3}$ have higher $e$ than the equilibrium configuration at $\mathrm{H}$, the jump will be to $\mathrm{H}_{1}$ with a lower $e$ than the equilibrium configuration at $\mathrm{H}$, see Fig. 7. Thus, the rod jumps from the equilibrium configuration at $\mathrm{H}$ with $n=2$ and $\gamma>0$ to the equilibrium configuration at $\mathrm{H}_{1}$ with $n=1$ and $\gamma>0$. From $\mathrm{H}_{1}$, decreasing $f$ follows along the lines described in 1 .

5. The rod is in an equilibrium configuration with $n=3$ and $\gamma>0$ on the blue curve denoted by I in Fig. 6. Increasing $f$ will move to an equilibrium configuration higher up on the blue curve and an increase in $e$, see Fig. 7. Decreasing $f$ moves to an equilibrium configuration lower down on the blue curve and a decrease in $e$. We can continue decreasing $f$ to reach $\mathrm{J}$, where $f=f_{\alpha}^{(3)}$. Decreasing $f$ further the equilibrium configuration transitions from one with $n=3$ and $\gamma=0$ to one with $n=2$ and $\gamma>0$. The furthest we can decrease $f$ to is $f=f_{\min }^{(2)}$ at $\mathrm{H}$. Once at $\mathrm{H}$, decreasing $f$ proceeds as described in 4 .

6. The rod is in an equilibrium configuration with $n=3$ and $\gamma<-2 \alpha$ on the blue curve denoted by K in Fig. 6. Increasing $f$ will move to an equilibrium configuration higher up on the blue curve and an increase in $e$, see Fig. 7. Decreasing $f$ moves to an equilibrium configuration lower down on the blue curve and a decrease in $e$. Continuously decreasing $f$ will result in reaching the equilibrium configuration with $f=f_{\min }^{(3)}$ at L. Once at $\mathrm{L}$, attempting to decrease $f$ further will result in a jump. This time we have five choices to jump to: $\mathrm{L}_{1}-\mathrm{L}_{5}$. Since $\mathrm{L}_{1}, \mathrm{~L}_{2}$ and $\mathrm{L}_{4}$ have greater $e$ than $\mathrm{L}$, we can discount jumps to them. Both $\mathrm{L}_{3}$ and $\mathrm{L}_{5}$ have less $e$ than $\mathrm{L}$. If a jump releases the least amount of $e$, then the rod jumps to the equilibrium configuration $\mathrm{L}_{5}$ with $n=1$ and $\gamma<-2 \alpha$. If a jump releases the greatest amount of $e$, then the rod jumps to an equilibrium configuration $\mathrm{L}_{3}$ with $n=1$ and $\gamma>0$. From $\mathrm{L}_{3}$ and $\mathrm{L}_{5}$, decreasing $f$ follows along the lines described in 1 and 2 , respectively. Thus, in this scenario 2 transition paths are possible.

7. Finally consider the rod in an equilibrium configuration at one of the local $f$ minima, $f_{\min }^{(n)}$. Take $\mathrm{D}$ for example where $f=f_{\min }^{(1)}$ in Fig. 6. Decreasing $f$ will result in a jump to $\mathrm{D}_{1}$ as described in 2. If $f$ is increased the rod can move to some equilibrium configuration between $\mathrm{D}$ and $\mathrm{C}$ or D and $\mathrm{F}$. The rod is more likely to choose an equilibrium configuration between $\mathrm{D}$ and $\mathrm{F}$ because this equilibrium configuration will have a lower $e$ than an equilibrium configuration between $\mathrm{D}$ and $\mathrm{C}$, see Fig. 7. The situation is almost like a bifurcation. Indeed, as $\alpha \rightarrow 0$, Eq. (42) tells us that we do have a bifurcation since the local $f$ minima $f_{\min }^{(n)}$ become the critical forces $f_{\mathrm{c}}^{(n)}$ for buckling. 


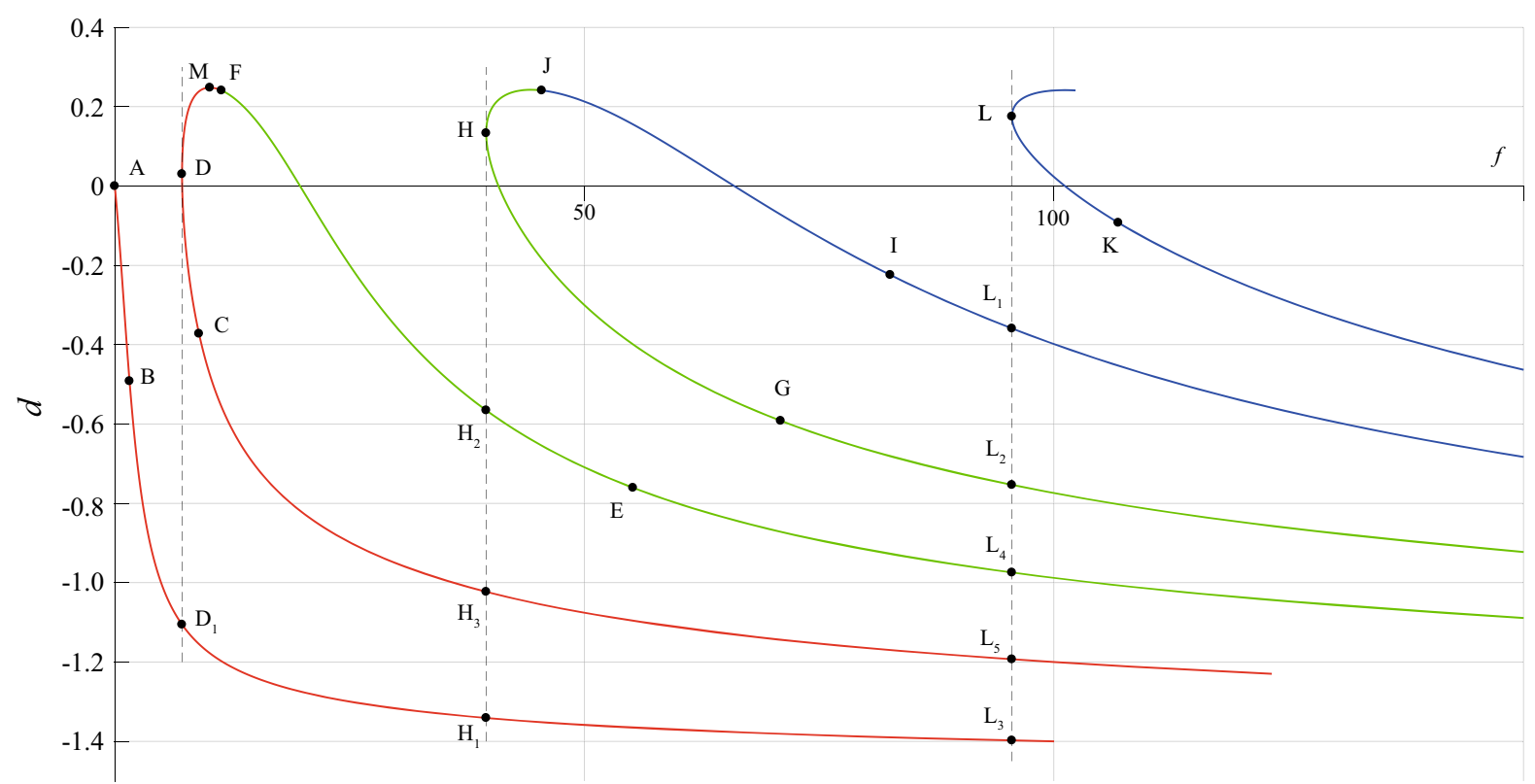

Fig. 8 A typical plot of $d$ against $f$ for equilibrium configurations with 1 (red), 2 (green) and 3 (blue) inflection points for $0<\alpha<\pi$. (Color figure online)

For equilibrium configurations with large $n$, multiple transitions paths may be possible during the process of decreasing $f$, i.e. unloading the rod.

The displacement of the free end of the rod in the direction of the inclined end force $f$ is denoted by $d$ where

$d=u(1)-\cos (\alpha)$.

Typical force-displacement $(f-d)$ plots for $0<\alpha<\pi$ are shown in Fig. 8. During the process of directly increasing and decreasing $f$ (dead loading), all arguments for scenarios 1-7 apply to the $f-d$ plots in Fig. 8. However, $f$ could also be provided indirectly by a device or mechanism that acts on the rod through some end displacement parameter $d$. That manner of providing $f$ is called rigid loading [6]. In that case, Eq. (47) is interpreted as a boundary condition for $u(1)$ that sets a constraint on the displacement of the free end of the rod in the direction of $f$. To specify the corresponding boundary value problem, the additional differential equation $\frac{\mathrm{d} f}{\mathrm{~d} s}=0$ is required, which ensures conservation of $f$ for each displacement $d$. Under that constraint, the rod can behave differently during loading and unloading. For example, consider scenario 3. The rod is in an equilibrium configuration with $n=1$ and $\gamma<-2 \alpha$ on the red curve denoted by $\mathrm{C}$ in Fig. 8. Indirectly decreasing $f$ by increasing $d$ moves to an equilibrium configuration higher up on the red curve and a decrease in $e$. Continually decreasing $f$ will result in reaching the equilibrium configuration with $f=f_{\min }^{(1)}$ at $\mathrm{D}$. However, there is still scope for increasing $d$ further until the local maximum (critical point) at $\mathrm{M}$ is reached. Once at $\mathrm{M}$, any further attempt to increase $d$ will result in a jump to A, the straight rod

\section{Conclusion}

The problem of the straight, inextensible and unshearable clamped-free elastic rod subjected to inclined end forces discussed in this article is well known $[1,2,4]$. Our contributions to the body of knowledge on this problem are as follows:

1. Exact solutions for all equilibrium configurations of the deformed rod in terms of elliptic integrals are given. Our version of the exact solutions makes the role of the number of inflection points in an inflectional equilibrium 
configuration central to their description. We also present explicit formulae for the arc length parameter $s$ and coordinates $u, v$ at those inflection points.

2. A discussion of critical points on $f-k$ plots has been presented and the locations of these critical points are determined. We identify critical points associated with local minima and maxima for $k$ as places where transitions between equilibrium configurations with $n$ and $n-1$ inflection points occur for $n \geq 2$. For critical points associated with local minima for $f$, a condition for locating the local force minimum $f_{\min }^{(\bar{n})}$ on the $n$th equilibrium configuration of the rod is presented.

3. General formulae are given for the number of equilibrium configurations for a given inclined end force $f$.

4. Applying arguments based on the elastic strain energy of the rod, we discuss scenarios where highly inflectional equilibrium configurations can transition to equilibrium configurations with fewer inflection points, and ultimately to the equilibrium configuration representing the straight rod.

5. It is pointed out that transitions between equilibrium configurations under rigid loading may differ from those under dead loading.

Acknowledgements This research was funded by London South Bank University. We would like to thank the reviewers for valuable comments and suggestions.

Open Access This article is distributed under the terms of the Creative Commons Attribution 4.0 International License (http:// creativecommons.org/licenses/by/4.0/), which permits unrestricted use, distribution, and reproduction in any medium, provided you give appropriate credit to the original author(s) and the source, provide a link to the Creative Commons license, and indicate if changes were made.

\section{References}

1. Navaee S, Elling RE (1992) Equilibrium configurations of cantilever beams subjected to inclined end loads. J Appl Mech 59(3):572579

2. Batista M (2014) Analytical treatment of equilibrium configurations of cantilever under terminal loads using jacobi elliptical functions. Int J Solids Struct 51(13):2308-2326

3. Timoshenko SP, Gere JM (1961) Theory of elastic stability, 2nd edn. McGraw-Hill Book Co, New York

4. Navaee S, Elling RE (1993) Possible ranges of end slope for cantilever beams. J Eng Mech 119(3):630-635

5. Batista M (2015) A simplified method to investigate the stability of cantilever rod equilibrium forms. Mech Res Commun 67:13-17

6. Thompson JMT (1961) Stability of elastic structures and their loading devices. J Mech Eng Sci 3(2):153-162

7. Antman SS (1995) Nonlinear problems of elasticity, 2nd edn. Springer, New York, pp 217-218

8. Audoly B, Pomeau Y (2010) Elasticity and geometry: from hair curls to the non-linear response of shells. Oxford University Press, Oxford, pp 114-120

9. Frisch-Fay R (1962) Flexible bars. Butterworths, London, pp 33-64

10. Dym CL, Shames IH (2013) Solid mechanics: a variational approach, augmented edn. Springer, New York, pp 516-521

11. Gradshteyn IS, Ryzhik IM (2007) Table of integrals, series, and products, 7th edn. Academic Press, San Diego, pp 859-883

12. Love AEH (1927) A treatise on the mathematical theory of elasticity, 4th edn. Cambridge University Press, Cambridge, pp 411-412

13. Bigoni D, Bosi F, Misseroni D, Dal Corso F, Noselli G (2015) New phenomena in nonlinear elastic structures: from tensile buckling to configurational forces. In: Bigoni D (ed) Extremely deformable structures. Springer, Vienna, pp 55-135

Publisher's Note Springer Nature remains neutral with regard to jurisdictional claims in published maps and institutional affiliations. 